$12 / 5.9580$

UCRL-ID-118721

\title{
Photonuclear Reactions in the GNASH Code: Benchmarking Model Calculations for Reactions on Lead up to $140 \mathrm{MeV}$
}

M. B. Chadwick

P. G. Young

August 1994

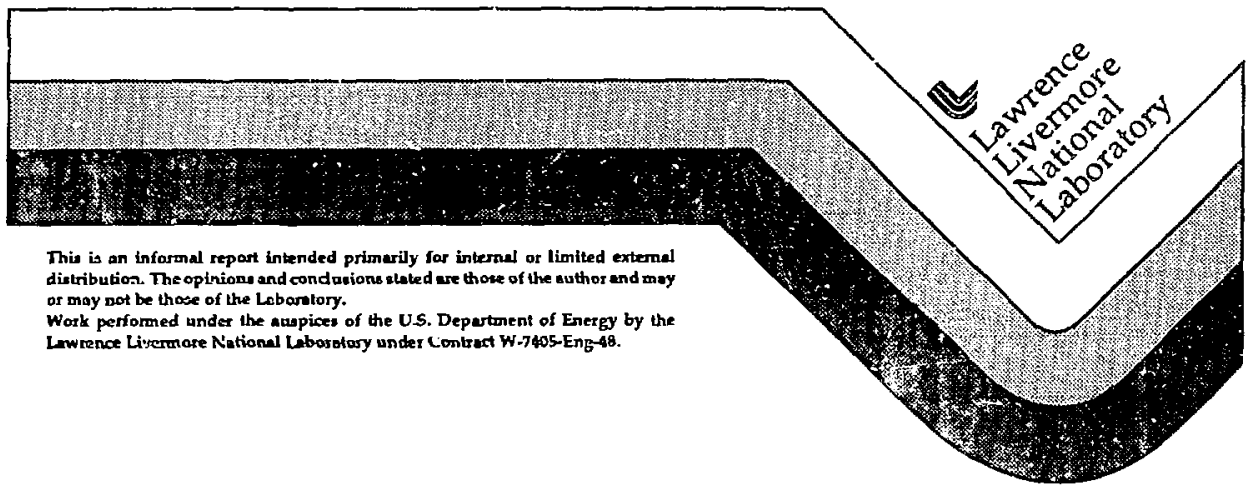




\section{DISCLAIMER}

This document was prepurce as an account of work sponsored by an agency of the United States Government. Nether the United Sestes Government nor the University of Califnrala bor any of their employees, makes any cran anty, express or tmpliv $\mathrm{d}$, ar escumes any legal liability or i esponsibility for the accuracy, completentess or usercitiess of ang information, appuraces, produch or process diselosed, or represents that its use would not Infringe privately ormed rights. Referense herein to any spedise conmerdal products, proces, or service by trade nome, trademark, manufactures, or otherwise, does not necsosarily constitute or imply its endorsement, recommendation, or favoring by the United State Government or the Uaiveralty of Calfornla. The views and opinioas of authors expressed hereln do not necrsarily stute or reflect those of the United States Government er the Univerdity of Califormia, and shall nal be used for advertising or product encui sement purpases.

This report has betn reproduced directly from the best avallable copy.

A valable to DOE and DOE contractors fram the Otfice of Selentific and Technical Information P.O. Bux 62, Oak Ridge, TN 37831

Prices ovailable from (615) 576-\$401, FTS 626-8401

A valleble to the public from the

Nationst Technical Intormation Service

US. Department of Commerce S28S Port Rny al Rd. Springlield, YA 22161 


\title{
Photonuclear Reactions in the GNASH Code: Benchmarking Model Calculations for Reactions on Lead up to $140 \mathrm{MeV}$
}

\author{
M. B. Chadwick' and P. G. Young ${ }^{2}$ \\ 'Lawrence Livermore National Laboratory, Livermore, Ci: 94550, USA \\ ${ }^{2}$ Los Alamos National Labomitory, Los Alamos, NM 87545, USA
}

\begin{abstract}
We have developed the GNASH code to include photonuclear reactions for incident energies up to $140 \mathrm{MeV}$. Photoabsorption is modeled through the giant resonance at the lower energies, and the quasideuteron mechanism at the higher energies, and the angular momentum coupling of the incident photon to the target is properly accounted for. After the initial interaction, primary and multiple preequilibrium emission of fast particles can occur before compound nucleus decay from the equilibrated compound nucleus. The angular distributions from compound nucleus decay are taken as isotropic. and those from preequilibrium emission (which we obtain from a phase-space nodel which conserves monentum) are forward-peaked. To test the new modeling we apply the code to calculate photonuclear reactions on ${ }^{20 x} \mathrm{~Pb}$ for incident energies up to $140 \mathrm{MeV}$.
\end{abstract}

\section{i. INTRODUCTION}

libraries of pholonuclear cross sections are needed for a range of new applications including photon radiation therapy, where the impact of secondary re'utron: collowing photon interactions with structural and shielding materials iil the accrlerator must be assessed. In order to generate such cross section libraries, we have developed the GNASH code to include photonuclear reactions. This involves (1) modeling the preequilibrium emission of fast nucleons, along with their angular distribulions, and (2) modifying the angular momentum couplings appropriate for a spin-one. negative parity (E1) photon projectile. With this code it is possible to unravel the experittental measurements shown in compilations such as that of Dietrich and Berman [1] and determine emission spectra of secondary particles that are emitted.

In order to benchmark our new modeling developments we have andyzcd photonuclear reactions on lead up to $140 \mathrm{MeV}$, where there exists much experimental 
data. Lead is of practical interest as a material used for radiation shielding, and in addition we have extensively studied nucleon-inauced reactions on lead $[2,3]$ and have already established many of the input parameters that are needed $[2,3]$.

In this report we descrit $~ o u r$ modeling of photonuclear reactions, and compars our calculations with experimental measurements of excitation functions and multiplicities. We demonstrate that photonuclear data can now be reliably predicted using the GNASH code.

\section{MODEL DEVELOPMENTS}

\section{A. The photoabsorption mechanism}

For incident energies below about $30 \mathrm{MeV}$, the photon wavelength is comparable to the size of the nucleus and photoabsorption proceeds primarily through a collective excitation of nucleus - the giant dipole resonance (GDR) - where the neutron and proton distributions undergo a bulk oscillation against each other. At higher energies. the photon wavelength becomes smaller, and photoabsorption occurs primarily on neutron-proton pairs (quasideuterons), which have a dipole moment and can absorb an E1 photon. A parameter-free model for quasideuteron (QD) photoabsorption was developed by Chadwick et al. \{4\}. Here we follow that work and describe the total cross section for photoabsorption as a sum of two terms: (1) the GDR, often described by a Lorentzian shape; and (2) the QD cross section. Tluus, we have

$$
\sigma_{\gamma}\left(c_{\gamma}\right)=\sigma_{G D R}\left(c_{\gamma}\right)+\sigma_{Q D}\left(c_{\gamma}\right)
$$

We describe the GDR using Lorentzian parameters taken from the compilation of Dietrich and Berman [1]. The quasideuteron cross section is given by [4]

$$
\sigma_{Q D}\left(c_{r}\right)=L \frac{N Z}{A} \sigma_{d}\left(c_{r}\right) f\left(c_{r}\right)
$$

where $L$ is the levinger parameter (derived to be 6.5$)$, and $f\left(c_{\gamma}\right)$ is the Pauli-bloxting function. which reduces the free deuteron cross setion $\sigma_{d}\left(c_{\gamma}\right)$ to account for Pauli. blocking of the excited neutron and proton by the nuclear medium. The experimental deuteron photodisintegration cross section was parameterized as $\sigma_{d}\left(c_{\gamma}\right)=$ $61.2\left(\epsilon_{\gamma}-2.224\right)^{3 / 2} / \epsilon_{\gamma}^{3} \mathrm{mb}$. The Pauli-blocking was derived by Chadwick et al. to be a multidimensional integral whose solution could be well approximated in the energy range $20-140 \mathrm{MeV}$ by the polynomial expression

$$
\begin{aligned}
& f\left(c_{\gamma}\right)=8.3714 \times 10^{-2}-9.8343 \times 10^{-3} \epsilon_{\gamma}+4.1222 \times 10^{-4} \epsilon_{\gamma}^{2} \\
&-3.4762 \times 10^{-6} \epsilon_{\gamma}^{3}+9.3537 \times 10^{-3} \epsilon_{\gamma}^{4} .
\end{aligned}
$$


In $\operatorname{Re}[.[4]$ the Pauli-blocking furction was not parameterized below $20 \mathrm{MeV}$, whcre the quasi-deuteron cross section becomes very small. Still, as the contribution needs to be defined at all energies considered, we use an exponential shape $f\left(c_{\gamma}\right)=\exp \left(-73.3 / c_{\gamma}\right)$ for energirs below $20 \mathrm{MeV}$. This form has the correct behavior in that it tends to zero at $\epsilon_{\gamma}=0$ and is continuous with Eq. (3) at $20 \mathrm{MeV}$.

Even though experimental data for the total photoabsorption cross sections tend to be well described by the sum of a Lorentzian and a QD contribution, as in Eq. (1), there are cases where this description fails, especially for light nuclei where ruclear structure effects strongly modify the shape of the total cross section data as a function of incident energy. Thus we have also included an option to perform the calculation with a previously-evaluated total photoabsorption cross section from an input file.

\section{B. Precquilibrium Emission}

Particle and hole excitations are produced in the nucleus following photoabsorption. Particle emission can occur from such a state yielding the typically highenergy preequilibrium emission; or alternatively, a nucleon-nucleon interaction may occur producing more particle-hole excitations. The composite nuclear system passes through such stages of increasing complexity towards equilibrium, and the total preequilibrium emission is the sum of the contributions from all the preequilibrium stages.

Wu and Change [5] and Blann [6] have successfully applied preequilibrium plysics modeling to describe the emission of high-energy nucleons following photoabsorption. Wu and Chang used an initial $2 p 2 h$ state in the preequilibrium cascade, while Blann argued that the two holes are corrclated through the QD mechanisn, and herefore should be thought of as one degree of froedom, i.e. a $2 p l h$ initial state. We follow Blann's prescription, and use the exciton model in GNASH to calculate the prcequilibrium emission of fast nucleons.

Once the incident photon energy exceeds about $50 \mathrm{MeV}$, multiple preequilibrium emission (MPE) becomes important, where more than one fast particle is ernitted. For this we use the generalized MPE model of Chadwick at al. [7], modified to reflect Lhe initial particle-hole type appropriate for the QD mechanism (i.e. of the two partirles. one is a neutron and the other a proton). Thus, if the first preequilibrium particle emited from the first $2 p 1 h$ stage is a neutron, the second preequilibrium particle must be a proton.

As in nuckon-induced reactions, we expect the angular distribution of prequilibrium nucleons to be forward-peaked since the incident projectile's energy and momentum are shared among the particle and hole degrees of freedom, and momentum conservation leads to a higher probability of emission in the forward direction. But since the momientum of a photon is considerably smaller than that of a nucleon for the same energy, the degree of forward peaking will be smaller for photonuclear reactions. It is not possible to use the phenomenological Kalbach systematics for the angular distributions since Kalbach did not consider photonuclear reactions, and very 
little experimental data exists for mono-energetic photon induced reactions. However, Chadwick and Oblozinsky [8] recently derived a theory for continuum angular distributions which provides a physical basis for the Kalbach systematics. This model, which uses state densities with linear momentum to obtain preequilibrium angular distributions, can be applied to obtain the angular distributions of the fast particles. From the preequilibriur.1 slage characterized by $n$ excitons the angular distribution is given by an exponential in $\cos \theta$,

$$
G(n, \theta)=\frac{1}{4 \pi} \frac{2 a}{e^{a}-e^{-a}} \exp (a \cos \theta) .
$$

The a-parameter governs the degree of forward peaking and is given by

$$
a=\frac{3 k k_{\Omega}}{2 n_{r} m \epsilon_{n v}}
$$

where $K$ is the incident photon momentum, $k_{\Omega}$ is the ernitted nucleon momentum relative to the bottom of the nuclear well, $n_{r}$ is the number of excitons in the residual nucleus, and $m$ is the nucleon mass. $\epsilon_{\Delta v}$ is the average exciton energy relative to the bottom of the well as given in Ref. [8].

\section{Compound nucleus decay}

We have modified GNASH so that it can describe the correct angular momentum and parity disiributions following photoabsorption. We assume that photoabsorption proceeds via the dominant electric-dipole interaction. Our work differs from previous calculations, which do not include angular momentum considerations. This improvement is likely to have a significant impact in the description of observables sensitive to angular momentum effects, i.e. isomer production cross sections and the cross sections of discrete gamma-ray lines in the residual nuclei that are activated.

Following preequilibrium emission, GNASH describes an open-ended sequencr of sequential compound nucleus emissions until all the energy brought in has been exlausted by either particle or gamma-ray' decay.

\section{RESULTS}

In our calculations we use the optical model and level density parameters. described in Ref. [2], to determine the particle emission rates. Wie compare our model calculations for lead with experimental data measured at Saclay. Lepretre et al. [9] obtained excitation functions for ${ }^{208} \mathrm{~Pb}(\gamma, \mathrm{xn})$ reactions, for incident photon energies up to $140 \mathrm{MeV}^{*}$. In Figs. J and 2 we show these excitation functions for $\mathrm{x}=1-1 \mathrm{l}$, which describe cross sections for emission of more or equal to $\mathrm{x}$ neutrons, compared with our model calculations. The good agreement with measurements supports our modeling of this reaction. The level of agreement that we obtain is comparable to that obtained by Blann using the ALICE code [6]. 
In Fig. 3 we show our calculated multiplicities for particle emission compared with the measurements of Lepretre et al. [10]. The upper figure shows the average neutron multiplicity, which is well described by our calculations. The lower figure shows the measured fast and slow multiplicitics at an incident photon energy of 70 $\mathrm{MeV}$, compared with our calculations. These measurements are invaluable for testing the preequilibrium modeling in our calculation, since direct measurements of the nucleon emission spectra from monoenergetic photons do not exist for lead. The fast multiplicity refers to the preequilibrium particles, while the slow multiplicity refers to the equilibrium (compound nucleus) particles, and our calculations are seen to describe the correct partitioning of ejectiles among preequilibrium and equilibrium emission, and between neutrons and protons. The large coulomb barrier in lead is responsible for the excess of fast preequilibrium neutrons compared to protons; at the highest energies the differences are reduced. In general the slow neutron multiplicity is much larger than the fast multiplicities, since preequilibrium decay accounts for at most the first two emissions, with the subsequent sequential particle decays coming from compound-nucleus emission. Slow compound-nucleus proton decay is negligible because of the coulomb barrier.

For the lower photon energies, experimental measurements are summarized in the Livermore compilation of Dietrich and Berman [1]. In a later work, Berman et al. concluded [II] that the earlier Livermore measurements on lead were too low, and therefore here we compare our calculations with the Saclay experiments. In Figs. 4-8 we show the measured total photoneutron cross section, the ${ }^{208} \mathrm{~Pb}(\gamma, 1 \mathrm{n}),{ }^{208} \mathrm{~Pb}\left(\gamma,{ }_{2}\right)$, the ${ }^{20 \mathrm{~S}} \mathrm{~Pb}(;, 3 \mathrm{n})$. and the phoioneutron yield cross sections compared with our calculations. Again. witb the exception of the ${ }^{208} \mathrm{~Pb}(\gamma, 2 \mathrm{n})$ reaction (which we overestinate. and which therefore also leads to an overprediction of the yield cross section between 15 and $20 \mathrm{MeV}$ ), our calculations describe the measurements well. Our calculations all use default input parameters, obtained in our other analyses of nucleon-induced reactions [2.3], and it is possible that by modifying these parameters we will iniprove the ${ }^{208} \mathrm{~Pb}(\gamma .2 \mathrm{n})$ calculation. The discrepancy here is related to competition between the $(\gamma, 1 n)$ and the $(\gamma, 2 n)$ reactions, and is sensitive to the level densities and paring energies used for ${ }^{208} \mathrm{~Pb}$ and ${ }^{207} \mathrm{~Pb}$.

In Fig. 9 we show our calculated double-differential neutron enission spertra for an incident pnoton energy of $140 \mathrm{MeV}$. At the low emission energies the emission is seen to be isolropic, and becomes increasingly forward-peaked for the higher cmission energies where preequilibrium mechanisms dominate.

While pholoneutron emission spectra from mono-energetic photons on lcad do not exist, a measurement of the photoneutron spectrum at 67 degrees from bremsstrahlung photons was made at Rensselaer Polytechnic institute by Liaushal et al. [13]. This measurement was made by subracting photoneutron speactra resulting from two separate bearns: a bremsstrahlung beam with maximum energy of $85 \mathrm{MeV}$; and a bremsstahlung beam with maximum energy of $55 \mathrm{MeV}$. The resulting neutron "difference spectrum" is due to photon incident energies between 55 and 
$85 \mathrm{MeV}$ (see Kaushal's paper for the spectrum). For simplicity in Fig. 10 we compare this result with our calculations at $70 \mathrm{MeV}$, approximately the average incident photon energy.

\section{CONCLUSIONS}

We have shown that the photonuclear modeling in GNASH describes the available experimental data well up to photon energies of $140 \mathrm{MeV}$. As there have been no measurements of the emission spectra following monoenergetic photon-induced reactions, we have tested our calculations by comparing them against excitation functions and multiplicity measurements, and against measured photoneutron spectra from a bremsstrahlung beam.

A complication that we may face when modeling lighter nuclei is that in many cases there are no measurements of the total photonuclear cross section; only the total photoneutron cross section. For light and medium weight nuclei the influence of the coulomb barrier is smaller, and photoproton emission can be significant. In this case it may be necessary to use an iterative approach to establish the total photonuclear cross section, by fitting the measured photoneutron cross section.

We have shown that all the tools needed for the modeling of photonuclear reaction with GNASH are in place, and photonuclear cross section libraries can now be developed.

He thank M. Blinn for his comments on the approaches that can he used for modeling photon induced reactions, and S. Warshaw and S. Chakrasarty for uscful discussions, and for providing us with a data base of experimental photonuclear measurements on lead.

\section{DISCLAIMER}

This account of work sponsored by an agency of the United States This report was prepared as an account of work sponsored by an agenthereof, nor any of their Government. Neither the Unitod States Governmed, or assumes any legal liability or responsiemployees, niakes any wasranty, express or implied. or ansu information, apparatus, produch or bility for the aceuracy, completeness, or usefulnces of any infor privately owned rights. Referprocess disclosed, or represents that its use would not inf or service by trade name, trademurk, ence thercin to any spocilic commercial prodsar, process, or semity or imply its endorsement, recommanufacturer, or oxiverwise does not necessarily constifte of imy ageney thereot. The views mendation, of favoring by the United States Goret nocessarily state of ieflect those of the and opinions of authors expressed herein do not.

United States Government of any agency thereor. 


\section{REFERENCES}

[1] Dietrich and B. Berman, Atomic Data and Nuclear Data Tables 38, 199 (1988).

[2] P. G. Young and M. B. Chadwick, in Specialist Meeting on Intermediate Energy Nuclear Data, edited by P. Nagel, May 30 - June 1, 1994, Issy-les-Moulineaux, France (to be published by the NEA/OECD).

[3] H. Vonach, A. Pavlik, M. B. Chadwick, R. Hajght, R. Nelson, P. G. Young, submitted to Phys. Rev. C (1994).

[4] M. B. Chadwick, P. Oblozinsky, G. Reffo, and P. E. Hodgson, Phys. Rev. C 44. $814(1991)$.

[.5] J. R. Wu and C. C. Chang, Phys. Rev. C 16, 1812 (1977)

[6] Mi. Blann, B. L. Berman, and T. T. Komoto, Phys. Rev. C 28, 2286 (1983).

[7] M. B. Chadwick, P. G. Young, D. C. George, and Y. Watanabe, (in print) Phys. Rev. C. (1994); M. B. Chadwick and P. G. Young. to be issued as a Lawrence Livermore Document (1994).

[8] M. B. Chadwick and P. Oblozinsky, submitted to Phys. Rev. Lelters, 1994; Lawrence Livernore Document UCRL-JC-117172 (1994).

[9] A. Lepretre, H. Beil, R. Bergere, P. Carlos, J. Fagot, A. de Miniac, and A. Veyssiere, Nucl. Phys. A367, 237 (1981).

[10] A. Lepretre. H. Beil, R. Bergere. P. Carlos. J. Fagot. A. Veyssiere, and 1. Ha]pern, Xucl. Fhis. A390, 221 (1982).

[11] B.L. Berman et. al., Plyss. Rev. C 36, 1286 (1985).

[12] A. Veyssiere, H. Beil, R. Bergere, P. Carlos, A. Lepretre, and A. de Miniac, Nucl. Phys. A227, 513 (1974).

(13) N. Kaushal et al., Phys. Rev. 1751330 (1968). 


\section{FIGURES}

FIG. L. Exritation functions for $x$ or more neutrons in the reaction ${ }^{209} \mathrm{~Pb}(y, x n)$, for $x=1-6$. Our model calculations are compared with the Lepretre data [9]

FIG. 2. Excitation functions for $x$ or more neutrons in the reaction ${ }^{208} \mathrm{~Pb}(\gamma, x n)$, for $x=7-11$. Our mode] calculations are compared with the Lepretre data [9]

FIG. 3. The upper figure shows our calculated average neutron multiplicities compared with the measurements of Lepretre et al. [10], for the $\gamma+{ }^{208} \mathrm{~Pb}$ reaction. The lower figure shows measured fast and slow average multiplicities at $70 \mathrm{MeV}$, compared with our calculations.

FIG. 4. Calculated excitation function of the total photoneutron cross section in the $\gamma+{ }^{208} \mathrm{~Pb}$ reaction compared with data data $[12]$

FIG. 5. Calculated excitation function of the ${ }^{208} \mathrm{~Pb}(\gamma, 1 n)$ reaction compared with data [12]

FIG. 6. Calculated excitation function of the ${ }^{208} \mathrm{~Pb}(\gamma .2 n)$ reaction compared with data $[12]$

FIG. 7. Calculated excitation function of the ${ }^{200} \mathrm{~Pb}(9,3 \pi)$ reaction compared with data [12]

FIG. 8. Calculated excitation function of the photoneutron yield in the $\gamma+{ }^{208} \mathrm{~Pb}$ reaction compared witł data data [12]

FIG. 9. Model calculations of the photoneutron einission spectra at various angles in the ${ }^{208} \mathrm{~Pb}(\gamma, x n)$ reaction, for an incident energy of $140 \mathrm{MeV}$. 
FIG. 10. Model calculation of the 67 -degrees photoneutron spectra at $E_{7}=70 \mathrm{Mcl}$ compared to measuremenus of difference neutrons from a bremsstrahlung beam of photons with energies between 55 and $85 \mathrm{MeV}$ 


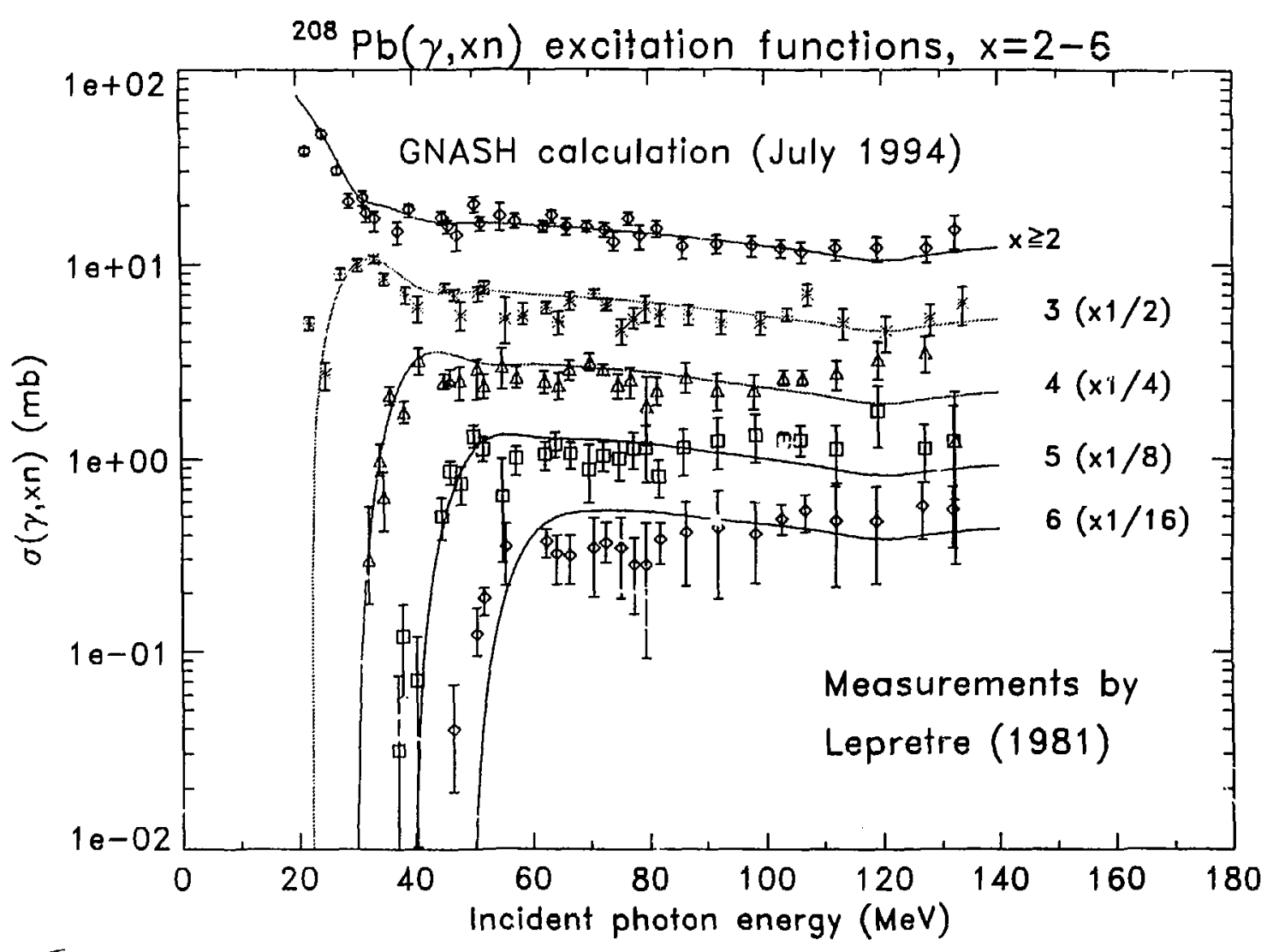

Fig $\geq 1$ 


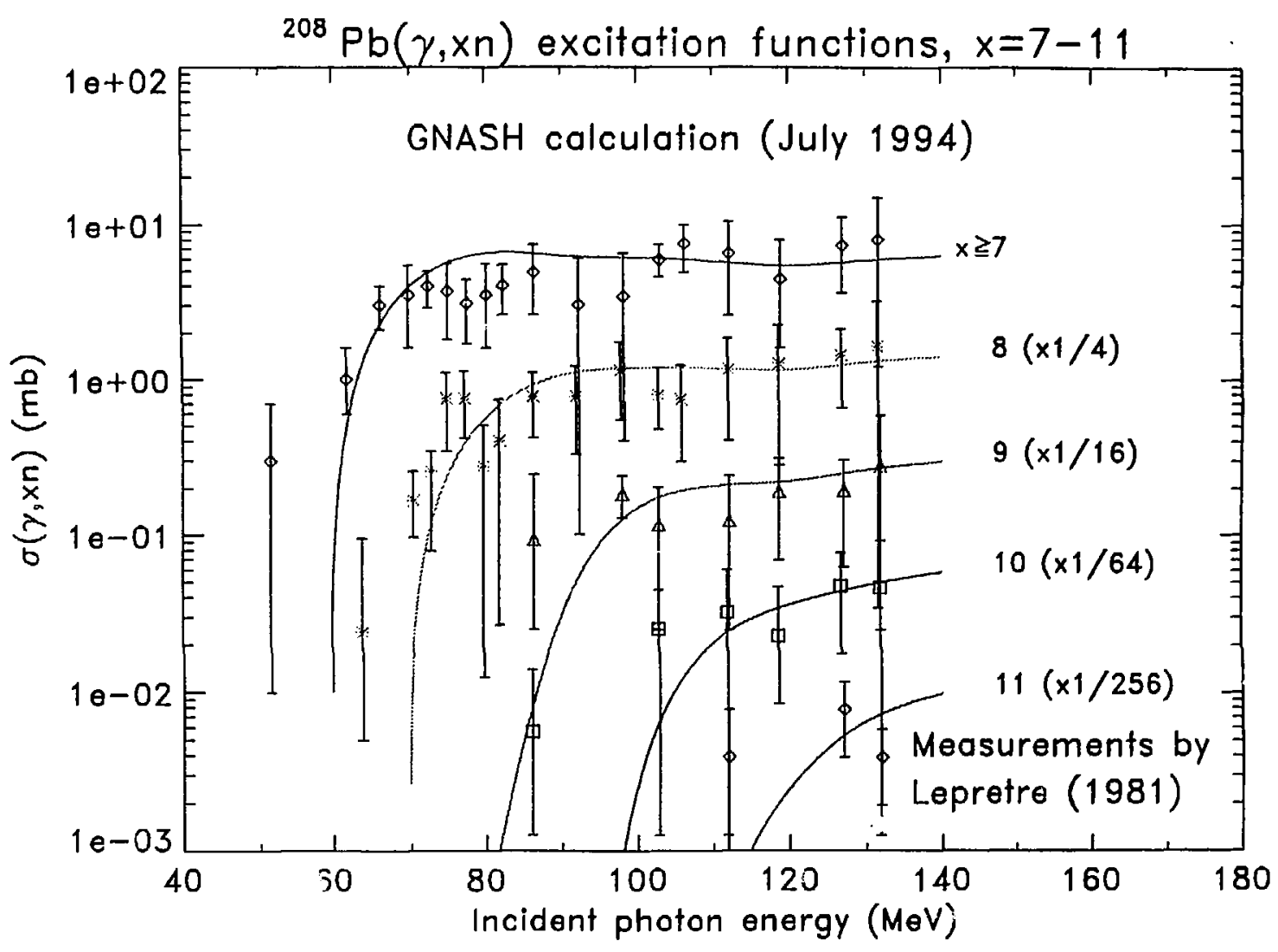

Fig 2 

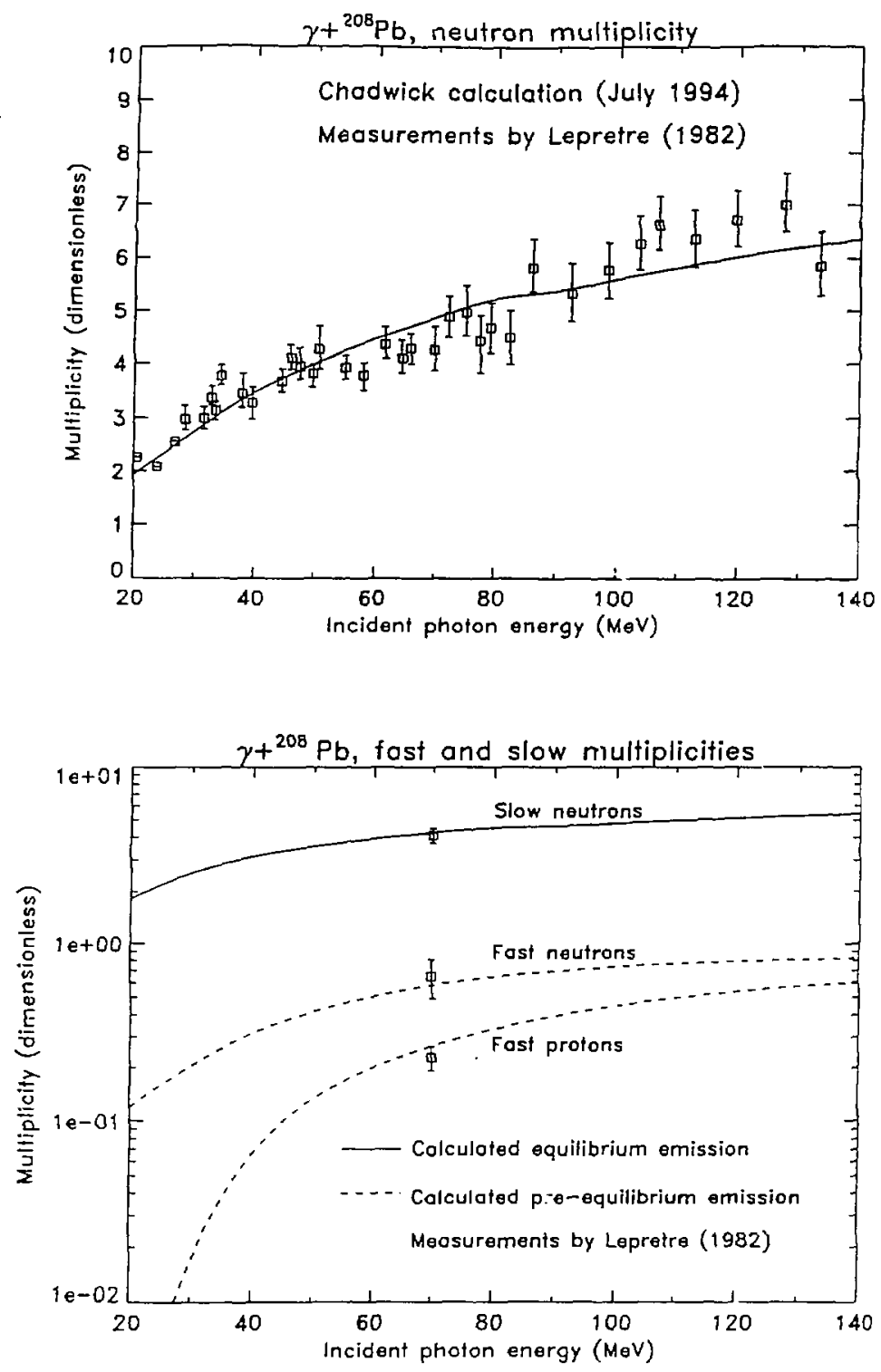

Fig 3 


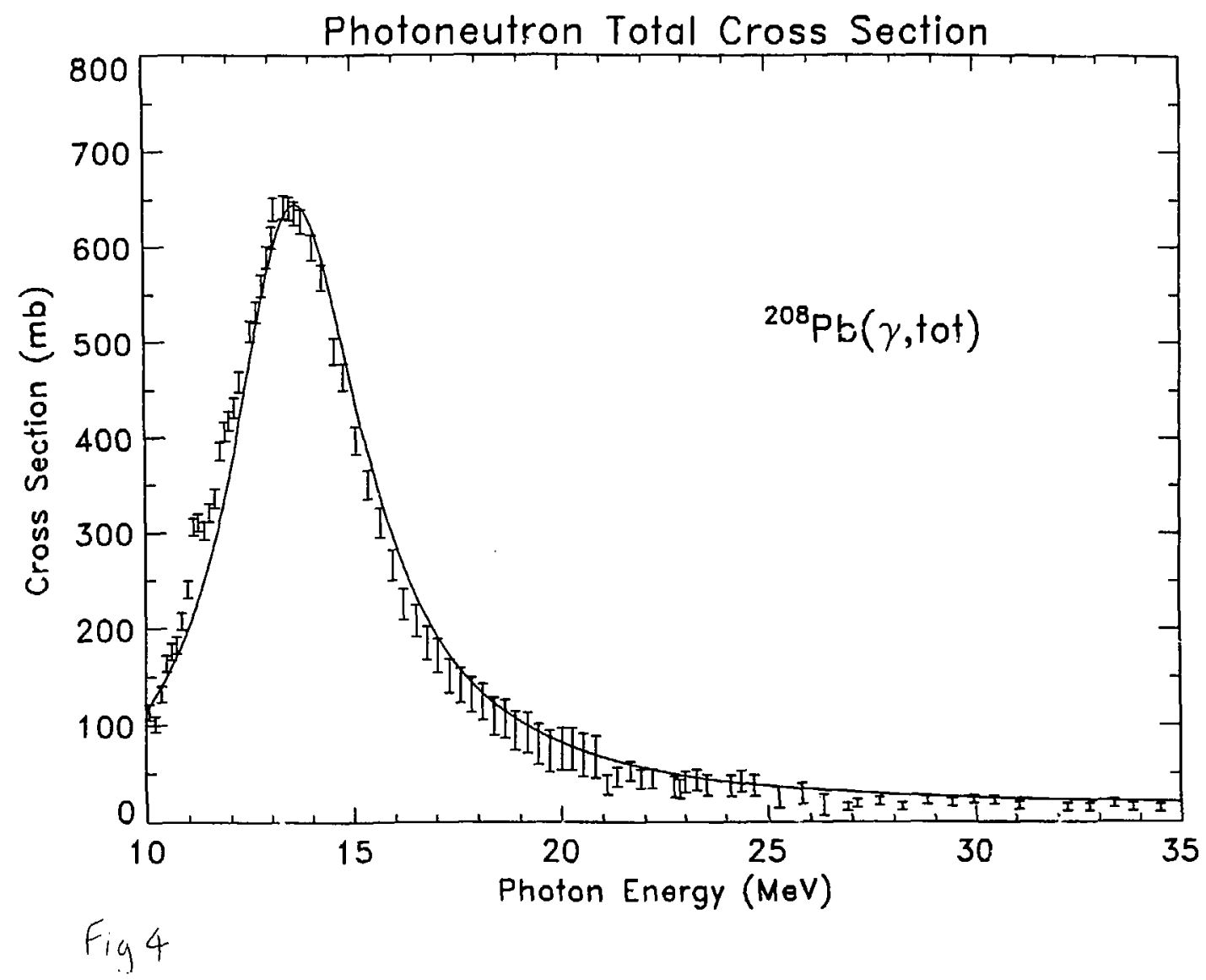


Single Photoneutron Cross Section

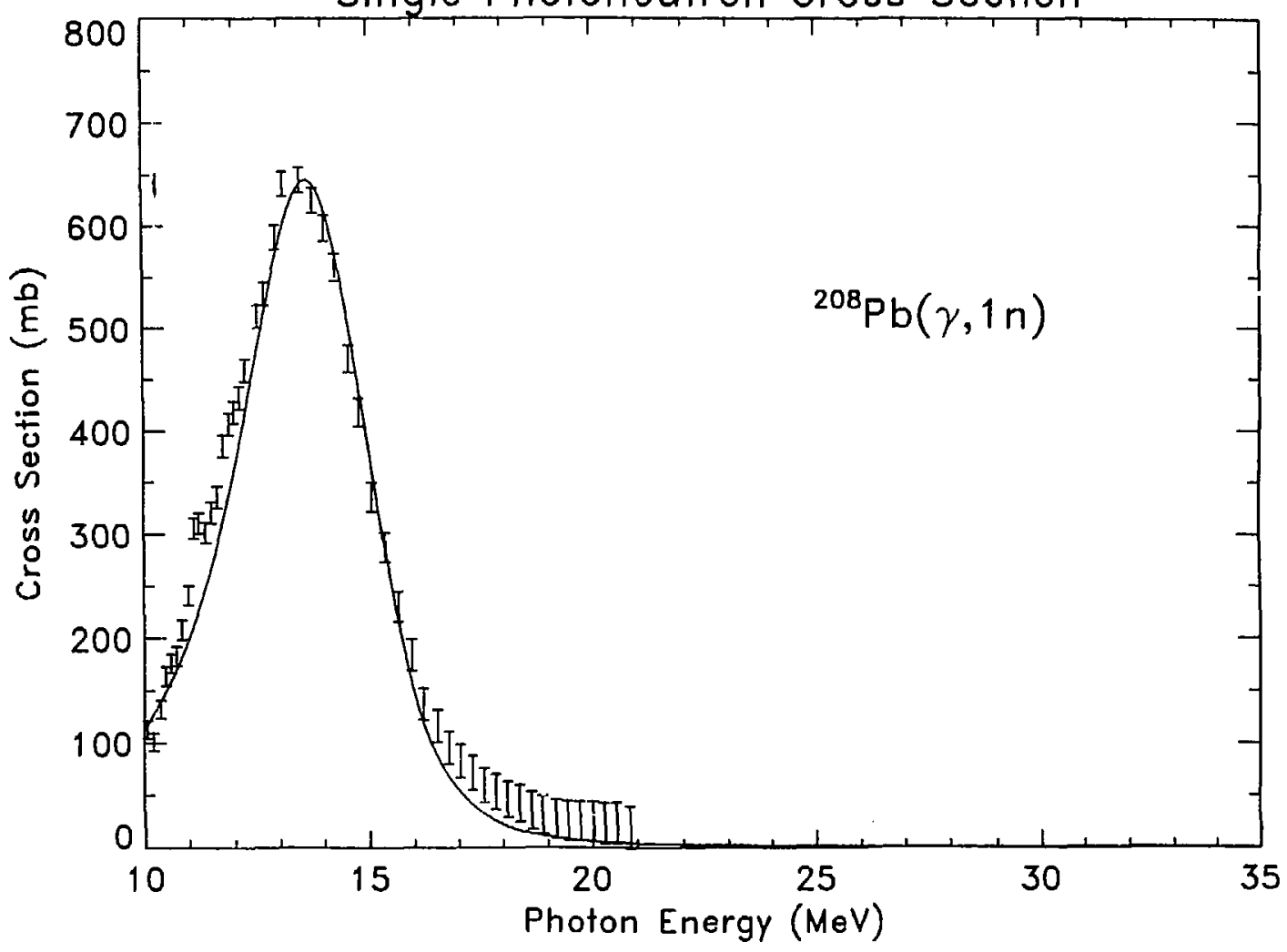

Fig 5 


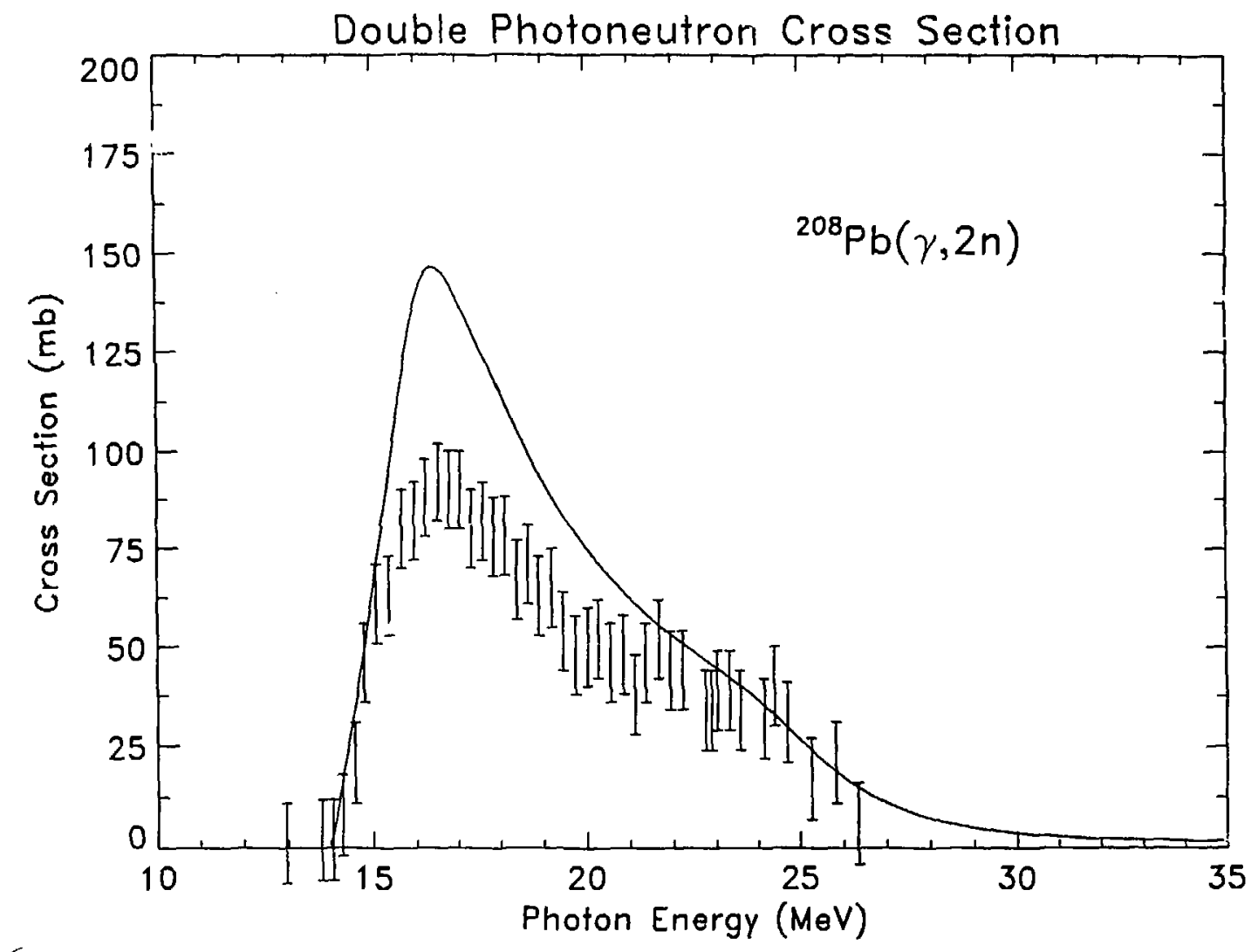

Fig 6 
Triple Photoneutron Cross Section

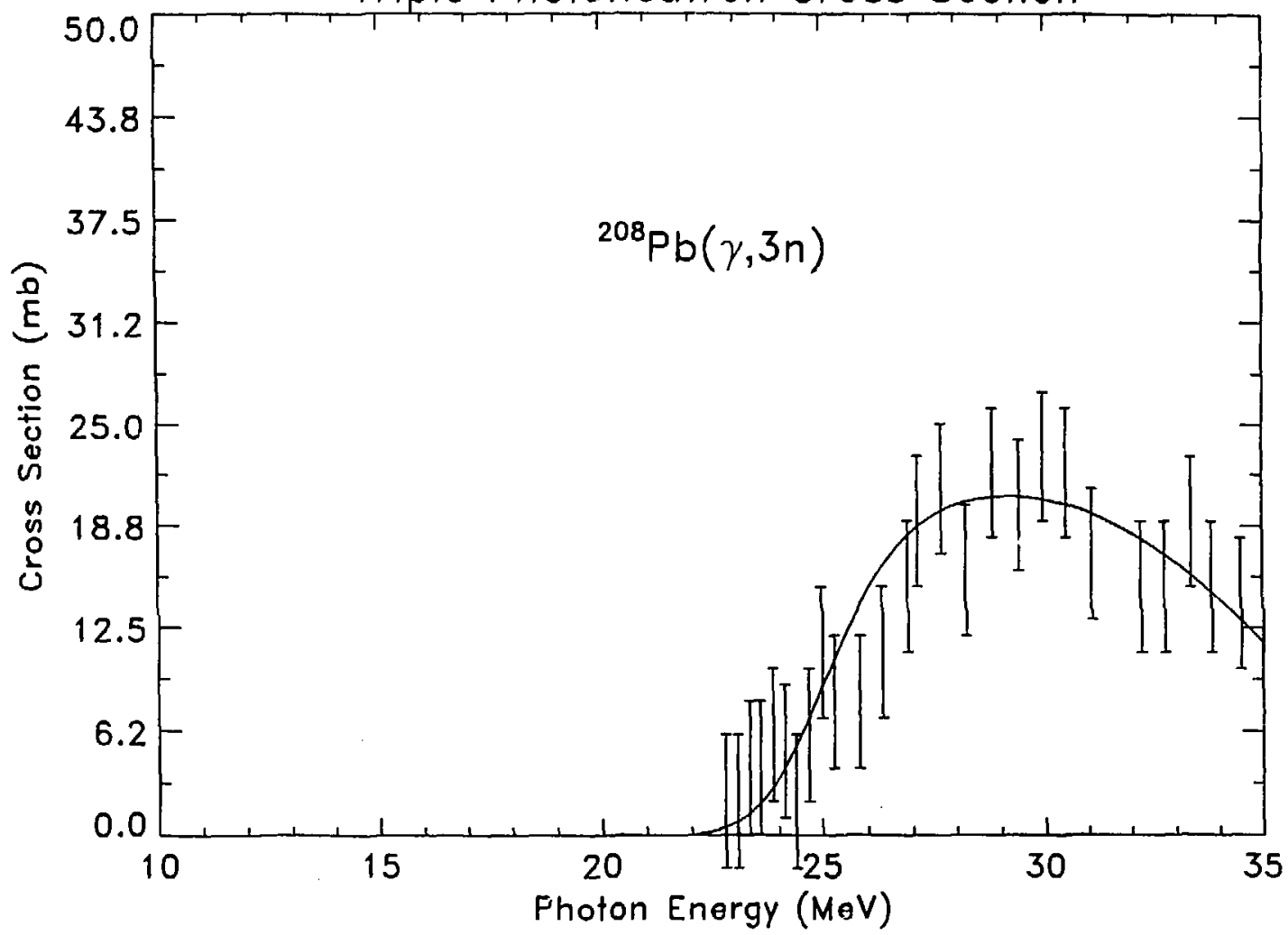

Fig 7 


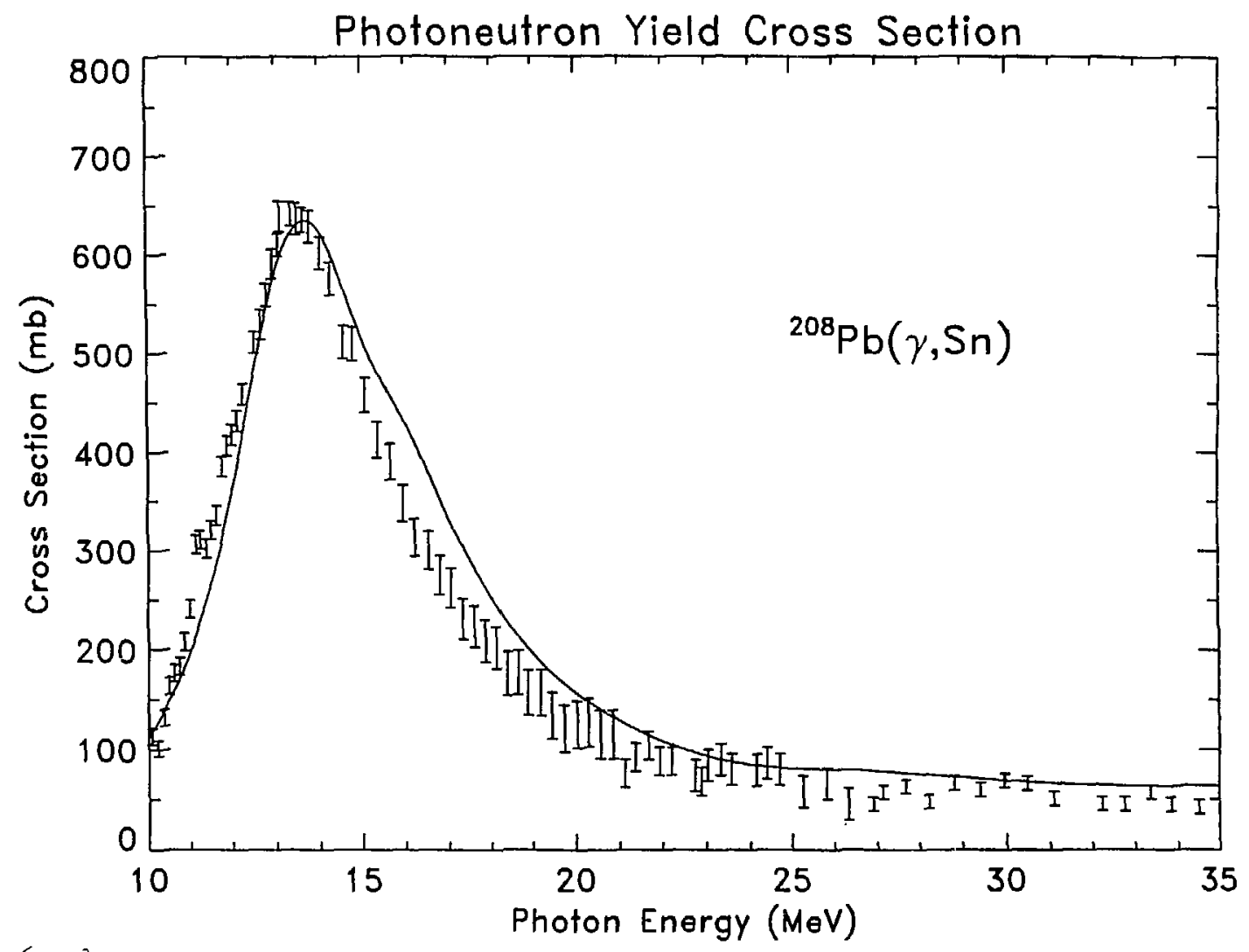

Fig 


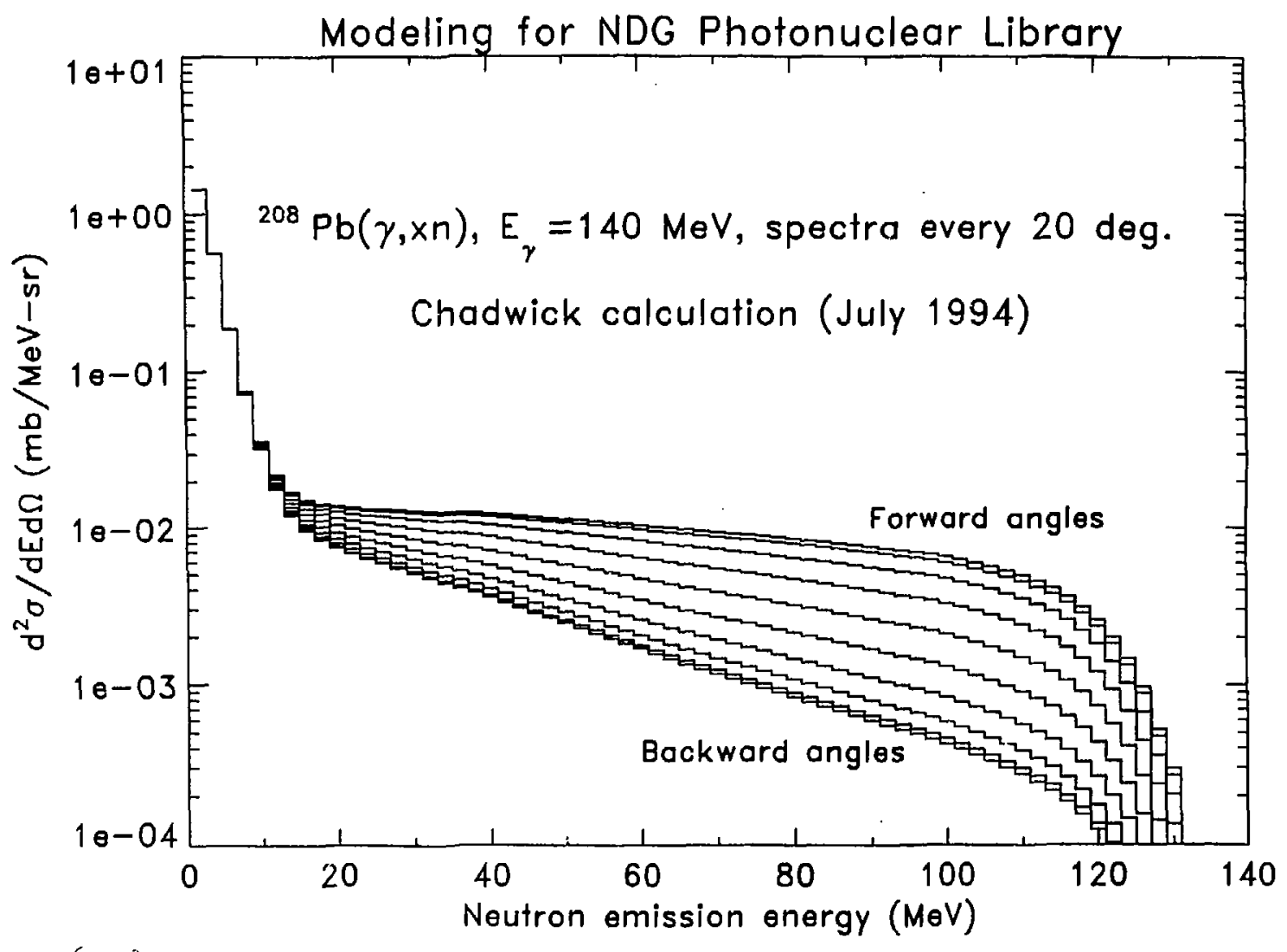

$f\left(g^{c}\right.$ 


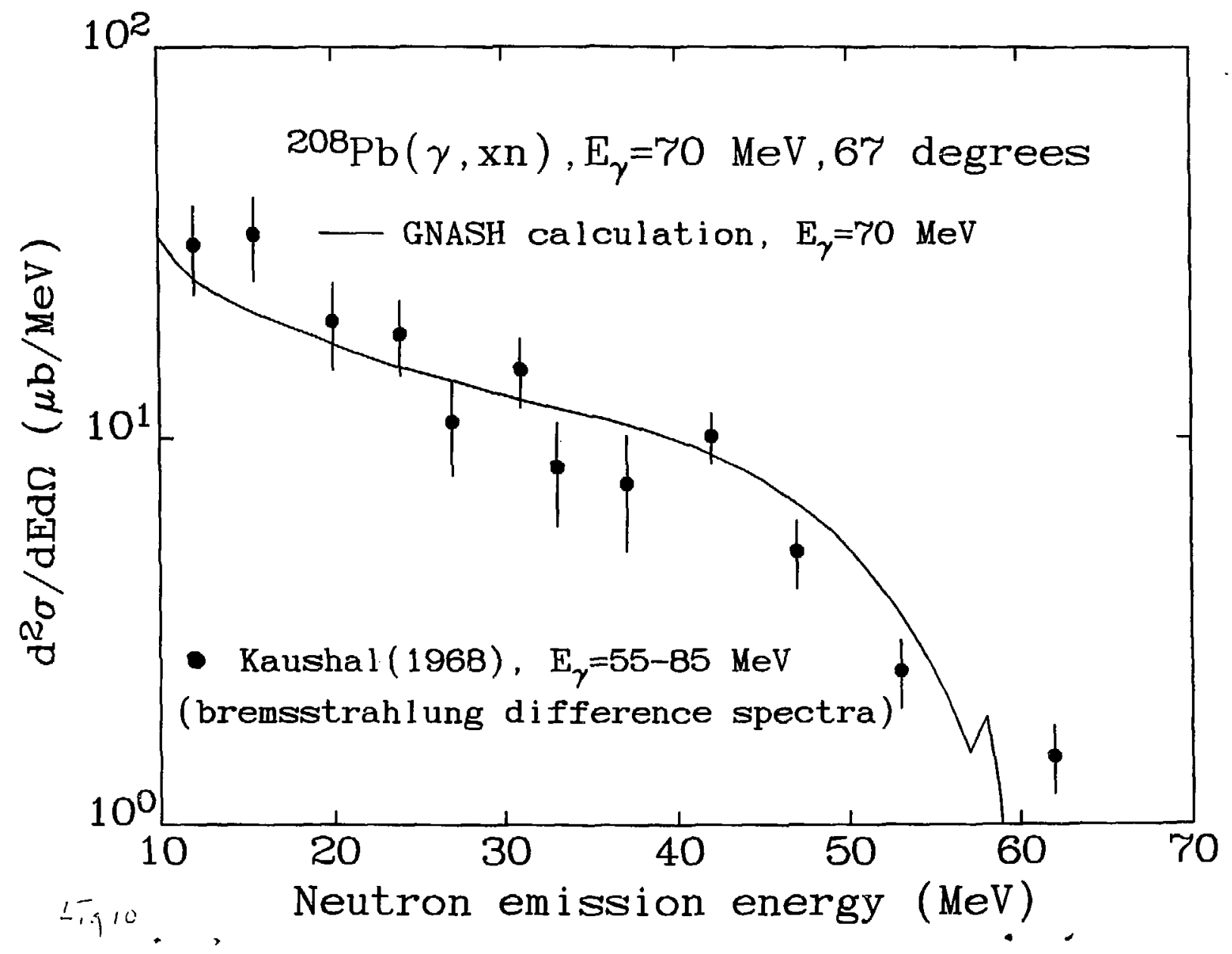

